This item was submitted to Loughborough's Research Repository by the author.

Items in Figshare are protected by copyright, with all rights reserved, unless otherwise indicated.

\title{
Durable high-performance water-based anti-reflective coating for PV module glass
}

PLEASE CITE THE PUBLISHED VERSION

https://doi.org/10.1109/PVSC.2018.8547886

PUBLISHER

(C) IEEE

VERSION

AM (Accepted Manuscript)

LICENCE

CC BY-NC-ND 4.0

\section{REPOSITORY RECORD}

Freiburger, Brennen M., Kenan Isbilir, Fabiana Lisco, Gerald Womack, Michael Walls, and Corey S. Thompson. 2019. "Durable High-performance Water-based Anti-reflective Coating for PV Module Glass". figshare. https://hdl.handle.net/2134/36947. 


\title{
Durable High-performance Water-based Anti-Reflective Coating for PV Module Glass
}

\author{
Brennen M. Freiburger ${ }^{1}$, Kenan Isbilir ${ }^{2},{\text { Fabina } \text { Lisco }^{2}, \text { Gerald Womack}^{2} \text {, John Michael Walls }}^{2}$, Corey S. \\ Thompson $^{1}$ \\ ${ }^{1}$ WattGlass, Inc., Fayetteville, AR, USA \\ ${ }^{2}$ CREST, Loughborough University, Leicestershire, UK
}

\begin{abstract}
Without an antireflective coating, more than $4 \%$ of incident light is reflected from the standard front cover glass of photovoltaic (PV) modules. Module efficiency is one of the most important levers to impact the cost-per-watt of solar and recovering some of this reflected light with a simple anti-reflective coating (ARC) has become widespread. The types of ARC can vary in deposition method (roll coating, spray coating, sputtering, etc.) as well as composition and performance. The most widely adopted coatings today are based on a porous silica film with a thickness optimized for the solar spectrum. Current coatings, however, have room for improvement in both the performance and cost, giving manufacturers reason to seek new solutions that drive down the levelized cost of electricity (LCOE). In this work, we report the test results for a new AR coating from WattGlass showing significantly improved optical performance compared to the traditional AR coatings. The WattGlass AR coating takes advantage of water-based chemistry that is more environmentally friendly than the sol-gel process used in standard production coatings. This chemistry allows a high performance and durable coating to be deposited and cured at room temperature and is compatible with an industry standard glass tempering process. The samples under test in this work were subjected to extensive environmental and accelerated lifetime testing performed by CREST (Centre for Renewable Energy Systems Technology) at Loughborough University.
\end{abstract}

Index Terms - antireflective coatings, module efficiency, photovoltaic cells.

\section{INTRODUCTION}

Due to the mismatch in index of refraction between air and glass, $4 \%$ of normally incident light is lost due to reflection at the interface of air and PV module glass. As the PV industry is driving towards higher efficiencies at even lower costs it has widely adopted the anti-reflective coating (ARC) for PV module front cover glass. It has been estimated that more than 92\% of silicon PV modules are now made with ARC glass [1].

Various types of ARCs are available, such as those obtained through various stacks of sputtered metal oxides, or chemical vapor deposited material. Porous silica coatings dominate the glass antireflective coating market due to their relative ease of deposition and the well-established sol-gel chemistries.
Most ARC glass manufacturers create the ARC on the solar cover glass by depositing a coating solution using techniques such as spray or roll coating. Sol-gels consisting of silica precursors (e.g., tetraethoxysilane, TEOS), acids, solvents, and water are ubiquitous in the solar ARC industry. The reaction of these components through the so-called Stöber process, creates silica that is deposited on the glass surface [2]. These coatings derive their optical and structural properties from their chemical composition and many require a temperaturecontrolled process step that occurs when the PV cover glass is tempered in order to reach a cured state. Porosity of the silica layer must be incorporated in some way to obtain the required optical properties of the anti-reflective coating layer. The method of forming this porosity is where the various ARC coatings are differentiated.

The porosity of these coatings has an inverse relationship with the index of refraction and is the mechanism for providing an index less than that of solid silica glass. This allows them to be used as quarter-wave thickness antireflective coatings [3]. As the index of refraction is further reduced to increase performance, an associated decrease in the mechanical strength of the coating due to the increased porosity is often observed [4]. This results in an inherent tradeoff between performance and durability of the coating.

The conversion efficiency of a cell/module is usually regarded as the most important performance parameter. However module manufactures and system owners put emphasis on the levelized cost of electricity (LCOE) which determines the financial return of a given project. As warranty periods extend, module manufactures are exploring coatings that couple long term reliability with improved performance.

In contrast to the commonly found, highly acidic, and solvent based sol-gel ARCs prevalent in the solar industry; the WattGlass AR coating, provides an environmentally friendly, completely water-based, high-performance ARC that is exceptionally durable and shelf-stable at room temperature. 


\section{EXPERIMENTAL DESIGN}

WattGlass prepared coated samples on $200 \mathrm{~mm}^{2} \times 3.2 \mathrm{~mm}$ Starphire low-iron glass and VWR VistaVision microscope slides as test substrates. Substrates were coated via dip coating followed by a brief heat treatment at $550^{\circ} \mathrm{C}$ in order to mimic tempering seen in the industrial scale process.

Samples were then provided to the CREST at Loughborough University, UK. The samples were characterized for optical performance by transmittance, reflectance, and spectroscopic ellipsometry. SEM imaging and water contact angle analysis were also conducted. In addition to characterization, samples were submitted to a battery of environmental and accelerated life testing based on IEC 61215-2:2016, including damp heat, UV exposure, thermal cycling, and humidity freeze. The coating was further exposed to mechanical abrasion, and sand impact tests adopted from IEC 1096-2 and ASTM D968-15, protocols respectively.

\section{RESULTS AND DISCUSSIONS}

\section{A. Optical Performance}

WattGlass AR coating showed a maximum increase in transmittance of $3.76 \%$ over that of the bare low-iron glass substrate and a measured reflectance of $4.8 \%$ at $550 \mathrm{~nm}$ wavelength for single-side coating. Weighted average transmittance and reflectance from $350-850 \mathrm{~nm}$ were $95.0 \%$ and 5\% respectively. Index of refraction dispersion and absorptance of the coating were determined through spectroscopic ellipsometry as shown in figure 1 .

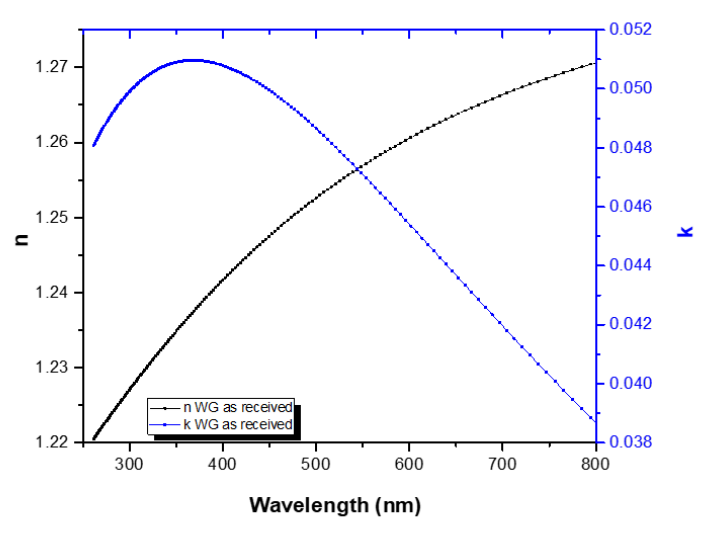

Fig. 1. Optical constants for WattGlass AR coating determined by spectroscopic ellipsometry

\section{B. Damp Heat Resistance.}

Damp heat resistance was evaluated by exposure to $85^{\circ} \mathrm{C}$ at $85 \%$ relative humidity $(\mathrm{RH})$. Intermediate checks of optical performance were performed between 50 and up to 1500 hours

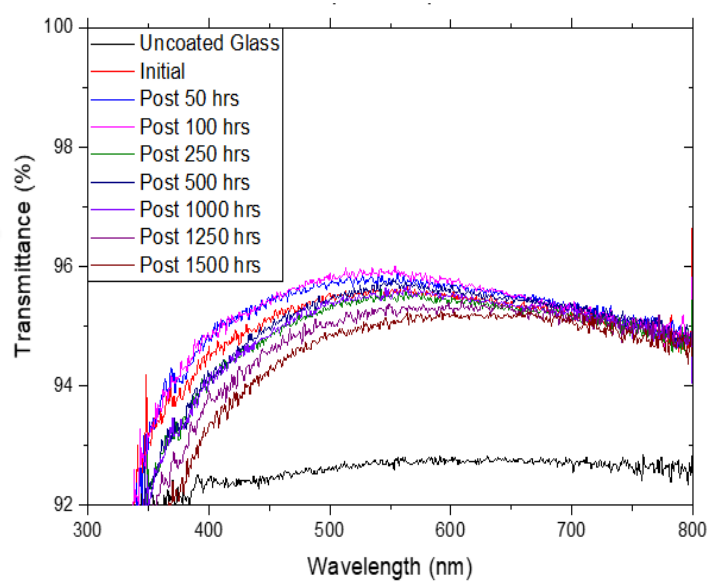

Fig. 2. Effect of damp heat exposure on optical transmittance of WattGlass AR coating from 0 to 1500 hours

of testing.

SEM and XPS analysis were performed following treatment of to 1000 hours and showed no significant changes. The effect on optical transmittance is shown in figure 2 .

\section{Abrasion Resistance}

The abrasion resistance of the coating was measured using various loads and a variety of abrasive materials on a linear abrader with $30 \mathrm{~mm}$ stroke length. The abrasive material used was a $175 \mathrm{~mm}^{2}$ felt pad at $5 \mathrm{~N}$ and $10 \mathrm{~N}$ force. The coating was

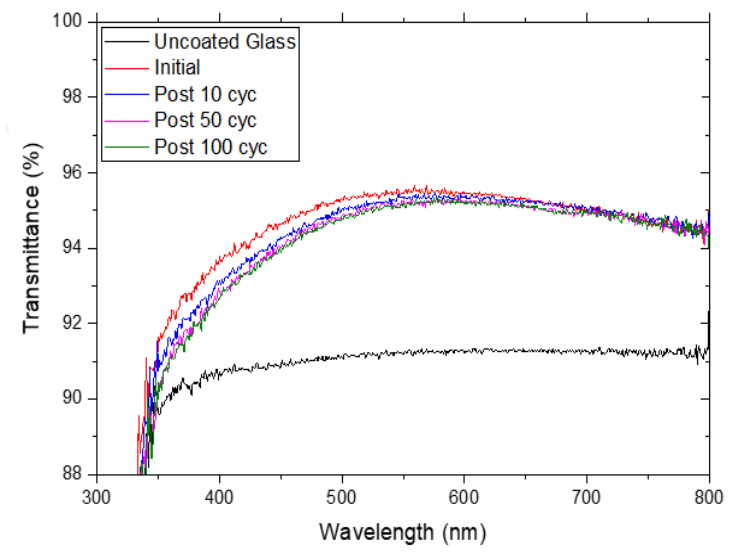

Fig. 3. Reduction in transmittance for abrasion treatment, 7.5 $\mathrm{mm}^{2}$ felt, $10 \mathrm{~N}$ force

subjected to a total of 100 cycles with intermediate checks at 10 and 50 cycles.

Tests reveal a maximum degradation in transmittance of $\sim 1 \%$ after a maximum stress condition of 100 cycles of felt pad at $10 \mathrm{~N}$ force as shown in figure 3. The main failure mechanism of the coating was revealed in optical micrograph to be scratching on the surface, which may be due to 


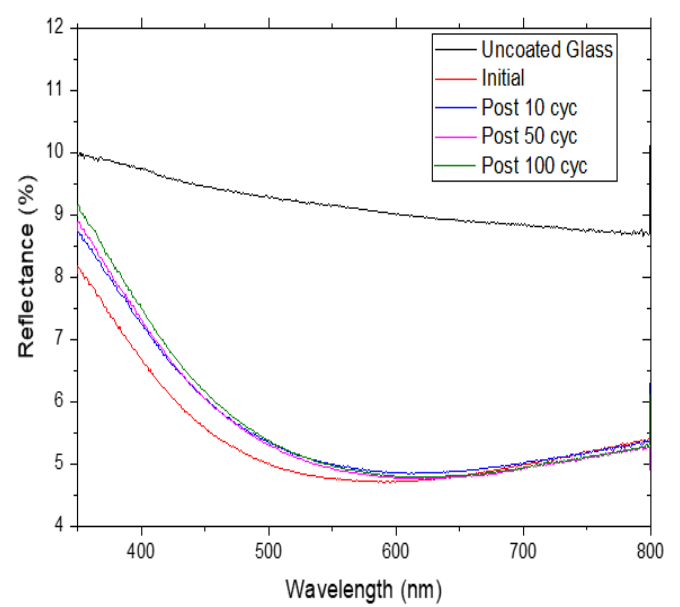

Fig. 4. Change in reflectance for abrasion treatment, $7.5 \mathrm{~mm}^{2}$ felt, $10 \mathrm{~N}$ force

particulate contamination of the felt counter face. These results will be followed with increasing the cycle count up to 400 and incorporation of CS8 and CS10 abrasive materials for comparison.

\section{Solubility}

Solubility was tested by immersion in DI water at room temperature, boiling DI water, or both. Cycling consisted of

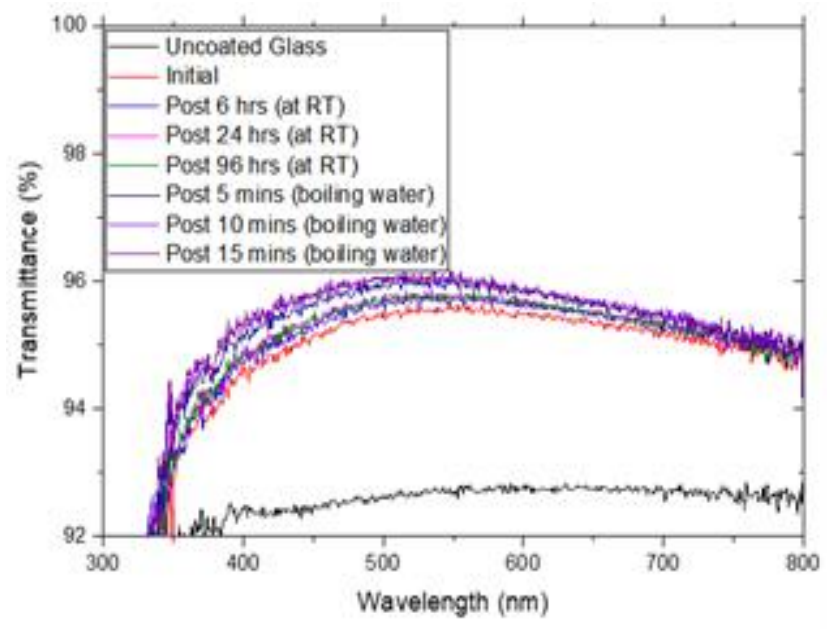

Fig. 5. Effect of boiling and room temperature (RT) water immersion tests on optical transmittance of WattGlass AR coating

immersion in boiling DI water for two minutes followed by immersion in DI water at room temperature for one minute. Results indicate the WattGlass AR coating is either unaffected or slightly improved by this treatment, as shown in figures 5 and 6.

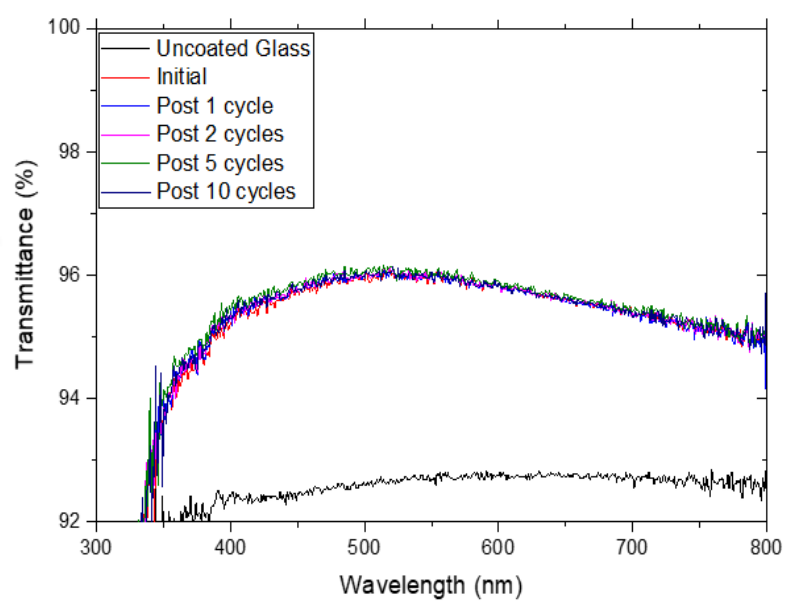

Fig. 6. Effect DI water immersion cycling tests on optical transmittance of WattGlass AR coating

\section{CONCLUSIONS}

In this evaluation, samples of the WattGlass AR coated glass were provided to CREST at Loughborough University for characterization and analysis. CREST conducted extensive characterization and testing for their optical performance and resistance to a variety of chemical, mechanical, and environmental stress tests. These results show that the WattGlass AR coating has the performance and durability to disrupt the solar ARC market. It is an economical, waterbased, and environmentally friendly solution that is a drop-in replacement for standard solvent and acid-based sol-gels currently prevalent in the industry.

The competing sol-gel coatings require harsh chemistries, expensive chemicals, and environmentally isolated processes, all impacting the cost to the manufacturer, the system owners, and the financial return of a given project. The combination of performance, robustness, and overall environmental conscientiousness of the WattGlass coatings make it a viable solution to increasing module efficiency through reduction of reflection at the PV cover glass interface.

\section{REFERENCES}

[1] International Technology Roadmap for Photovoltaics (ITRPV) 2015 Results, March 2016.

[2] D. Chen, "Anti-reflection (AR) coatings made by sol-gel processes: a review," Solar Energy Materials and Solar Cells, vol. 68, pp. 313-336, 2001.

[3] R. Prado, G. Beobide, A. Marcaide, J. Goikoetxea, A. Aranzabe, "Development of multifunctional sol-gel coatings: Anti- reflection coatings with enhanced self-cleaning capacity', Solar Energy Materials \& Solar Cells, vol. 94, pp. 1084-1088, 2010.

[4] S. C. Pop, et al., "A highly abrasive-resistant, long-lasting antireflective coating for PV module glass," in the $40^{\text {th }}$ IEEE Photovoltaic Specialists Conference, 2014. 